International Journal of Pure and Applied Mathematics

Volume 98 No. 2 2015, 181-192

ISSN: 1311-8080 (printed version); ISSN: 1314-3395 (on-line version)

url: http://www.ijpam.eu

doi: http://dx.doi.org/10.12732/ijpam.v98i2.2

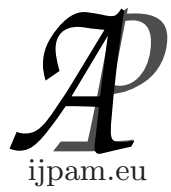

\title{
ON THE DIVISORS OF ORDER $r$
}

\author{
Andrew V. Lelechenko ${ }^{1}$, Yakov A. Vorobyov ${ }^{2}$ \\ ${ }^{1,2}$ Computational Algebra and Discrete Mathematics \\ Odessa National University \\ Dvoryanskaya st. 2, Odessa, 65026, UKRAINE
}

\begin{abstract}
N. Minculete has introduced the divisor-of-order- $r$ function $\tau^{(r)}$ and the sum-of-divisors-of-order- $r$ function $\sigma^{(r)}$. We investigate the asymptotic behaviour of $\sum_{n \leq x} \tau^{(r)}(n)$ and $\sum_{n \leq x} \sigma^{(r)}(n)$ and improve known estimates.

AMS Subject Classification: 11A25, 11N37

Key Words: divisor of order $r$, exponential semiproper divisor, average order
\end{abstract}

\section{Introduction}

Recently N. Minculete in his $\mathrm{PhD}$ Thesis [8] introduced a concept of divisors of order $r$ : integer $d=p_{1}^{b_{1}} \cdots p_{k}^{b_{k}}$ is called a divisor of order $r$ of number $n=p_{1}^{a_{1}} \cdots p_{k}^{a_{k}}$ if $d$ divides $n$ in the usual sense and $b_{j} \in\left\{r, a_{j}\right\}$ for $j=1, \ldots, k$. We also suppose that 1 is a divisor of any order of itself (but not of any other number). Let us denote respective divisor and sum-of-divisor functions as $\tau^{(r)}$ and $\sigma^{(r)}$. These functions are multiplicative and

$$
\begin{aligned}
\tau^{(r)}\left(p^{a}\right) & = \begin{cases}1, & a \leq r, \\
2, & a>r .\end{cases} \\
\sigma^{(r)}\left(p^{a}\right) & = \begin{cases}p^{a}, & a \leq r, \\
p^{a}+p^{r}, & a>r .\end{cases}
\end{aligned}
$$

In a special case of $r=0$ we get well-studied unitary divisors, see $[2,11]$. Below we consider only the case $r>0$.

Received: June 2, 2014

$\S_{\text {Correspondence author }}$ (c) 2015 Academic Publications, Ltd. url: www.acadpubl.eu 
In another special case of $r=1$ we get so-called by Minculete exponential semiproper divisors and denote $\tau^{(e) s}:=\tau^{(1)}, \sigma^{(e) s}:=\sigma^{(1)}$. An integer $d$ is an exponential semiproper divisor of $n$ if $\operatorname{ker} d=\operatorname{ker} n$ and $(d / \operatorname{ker} n, n / d)=1$, where $\operatorname{ker} n=\prod_{p \mid n} p$.

Minculete proved in $[8,(3.1 .17-19)]$ that

$$
\begin{gathered}
\limsup _{n \rightarrow \infty} \frac{\log \tau^{(r)}(n) \log \log n}{\log n}=\frac{\log 2}{r+1}, \\
\sum_{n \leq x} \tau^{(r)}(n)=\frac{\zeta(r+1)}{\zeta(2 r+2)} x+A x^{1 /(r+1)}+O\left(x^{1 /(r+2)+\varepsilon}\right), \\
\limsup _{n \rightarrow \infty} \frac{\sigma^{(r)}(n)}{n \log \log n}=\frac{6 e^{\gamma}}{\pi^{2}} .
\end{gathered}
$$

In the present paper we improve the error term in (4) and establish asymptotic formulas for $\sum_{n \leq x} \sigma^{(r)}(n)$ with $O$ - and $\Omega$-estimates of the error term.

In asymptotic relations we use $\sim, \asymp$, Landau symbols $O$ and $o$, big omegas $\Omega$ and $\Omega_{ \pm}$, Vinogradov symbols $\ll$ and $\gg$ in their usual meanings. All asymptotic relations are given as an argument tends to the infinity.

Letter $p$ with or without indexes denote rational prime.

As usual $\zeta(s)$ is the Riemann zeta-function. For complex $s$ we denote $\sigma:=$ $\Re s$ and $t:=\Im s$.

We use abbreviations $\log x:=\log \log x, \operatorname{lllog} x:=\log \log \log x$.

Letter $\gamma$ denotes Euler-Mascheroni constant, $\gamma \approx 0.577$.

Everywhere $\varepsilon>0$ is an arbitrarily small number (not always the same even in one equation).

We write $f \star g$ for Dirichlet convolution:

$$
(f \star g)(n)=\sum_{d \mid n} f(d) g(n / d) .
$$

Function ker: $\mathbf{N} \rightarrow \mathbf{N}$ stands for $\operatorname{ker} n=\prod_{p \mid n} p$.

For a set $A$ notation \#A means the cardinality of $A$.

\section{Preliminary Estimates}

Consider $\tau(a, b ; n)=\sum_{k^{a} l^{b}=n} 1$ and $T(a, b ; x)=\sum_{n \leq x} \tau(a, b ; n)$ for $1 \leq a \leq b$. One can directly check that $\sum_{n=1}^{\infty} \tau(a, b ; n) n^{-s}=\zeta(\overline{a s}) \zeta(b s)$ for $\sigma>1$. 
Lemma 1. $T(a, b ; x)=H(a, b ; x)+\Delta(a, b ; x)$, where

$$
\begin{gathered}
H(a, b ; x)= \begin{cases}\zeta(b / a) x^{1 / a}+\zeta(a / b) x^{1 / b}, & 1 \leq a<b, \\
x^{1 / a} \log x+(2 \gamma-1) x^{1 / a}, & a=b,\end{cases} \\
x^{1 / 2(a+b)} \ll \Delta(a, b ; x) \ll \begin{cases}x^{1 /(2 a+b)} & 1 \leq a<b, \\
x^{1 / 3 a} \log x & a=b .\end{cases}
\end{gathered}
$$

Proof. See [6, Th. 5.1, Th. 5.3, Th. 5.8].

In fact $\Delta(a, b ; x)$ can be estimated more precisely. For our goals we are primarily interested in the behaviour of $\Delta(1, b ; x)$. Let us suppose that

$$
\Delta(1, b ; x) \ll x^{\theta_{b}} \log ^{\theta_{b}^{\prime}} x,
$$

then due to [6, Th. 5.11] for $b \geq 7$ we can choose $\theta_{b}=1 /(b+7 / 2)$ and $\theta_{b}^{\prime}=1$. Estimates for $b \leq 16$ are given in Table 1 . Estimate for $b=1$ belongs to Huxley [4], and estimate for $b=2$ belongs to Graham and Kolesnik [3]. We have found no references on the best known results for $b \geq 3$, so we calculated them with the use of [6, Th. 5.11, Th. 5.12] selecting appropriate exponent pairs carefully. It seems that some of this estimates may be new.

Lemma 2. Let $\alpha$ and $\beta$ be positive real numbers with $\beta+1=\alpha$. Then

$$
\sum_{m n^{\alpha} \leq x} m n^{\beta}=\frac{\zeta(2 \alpha-\beta)}{2} x^{2}+\mathcal{D}(\alpha, \beta ; x), \quad \mathcal{D}(\alpha, \beta ; x) \ll x \log ^{2 / 3} x .
$$

Proof. See [10, Th. 1].

For $k>0$ one can define a multiplicative function $\mu_{k}$ implicitly by

$$
\sum_{n=1}^{\infty} \mu_{k}(n) n^{-s}=1 / \zeta(k s)
$$

for $\sigma>1$. So $\mu_{k}\left(n^{k}\right)=\mu(n)$ and $\mu_{k}(m)=0$ for all other arguments. Trivially $\mu_{1} \equiv \mu$. Then

$$
M_{k}(x):=\sum_{n \leq x} \mu_{k}(n)=\sum_{n \leq x^{1 / k}} \mu(n) \ll x^{1 / k} \exp (-C N(x)),
$$

where $C>0, N(x)=\log ^{3 / 5} x \operatorname{llog}^{-1 / 5} x$. See [5, Th. 12.7] for the proof of the last estimate. Assuming Riemann hypothesis $(\mathrm{RH})$ we get much better result $M_{k}(x) \ll x^{1 / 2 k+\varepsilon}[12$, Th. $14.25(\mathrm{C})]$. 


\begin{tabular}{r|r|c|c}
$b$ & $\theta_{b}$ & $\theta_{b}^{\prime}$ & Exponent pair or reference \\
\hline 1 & $131 / 416+\varepsilon \approx 0.314904$ & 0 & {$[4]$} \\
\hline 2 & $1057 / 4785+\varepsilon \approx 0.220899$ & 0 & {$[3]$} \\
\hline 3 & $1486 / 8647+\varepsilon \approx 0.171852$ & 0 & $A B(A \mathcal{B})^{2} H$ \\
\hline 4 & $\approx 0.140161$ & 0 & $A H$ \\
\hline 5 & $\approx 0.121398$ & 2 & $A^{2} \mathcal{B} A\left(A \mathcal{B} A \mathcal{B}^{2} A^{2} \mathcal{B}^{6}\right)^{\infty} I$ \\
\hline 6 & $669 / 6305 \approx 0.106106$ & 1 & $\left(A^{2} B\right)^{3}(A B)^{3} A^{4} B I$ \\
\hline 7 & $\approx 0.094491$ & 2 & $A^{2} \mathcal{B}^{2}\left(\mathcal{B} A \mathcal{B}^{2} A^{2} \mathcal{B}^{6} A\right)^{\infty} I$ \\
\hline 8 & $\approx 0.085314$ & 1 & $A^{2} \mathcal{B}^{4} A\left(A \mathcal{B} A^{2}\right)^{\infty} I$ \\
\hline 9 & $\approx 0.077892$ & 2 & $A^{2} \mathcal{B}^{2}\left(A \mathcal{B}^{6} A \mathcal{B} A \mathcal{B}^{2} A\right)^{\infty} I$ \\
\hline 10 & $\approx 0.071774$ & 0 & $\left(A^{2} \mathcal{B}^{2}\right)^{3} A \mathcal{B} H$ \\
\hline 11 & $\approx 0.066283$ & 0 & $A^{2} H$ \\
\hline 12 & $64 / 1037+\varepsilon \approx 0.061716$ & 0 & $A^{2} H$ \\
\hline 13 & $\approx 0.058089$ & 0 & $A^{3} B A^{3} B A^{2} B A^{4} B(A \mathcal{B})^{2} H$ \\
\hline 14 & $75 / 1373 \approx 0.054625$ & 1 & $A^{2}(A \mathcal{B})^{2} B A^{3} B I$ \\
\hline 15 & $\approx 0.051570$ & 0 & $A\left(A^{2} B\right)^{3} A^{4} \mathcal{B}^{7} A^{3} \mathcal{B} B A^{4} \mathcal{B} H$ \\
\hline 16 & $15 / 307 \approx 0.048860$ & 1 & $A^{3} B A^{2} B A^{4} B I$
\end{tabular}

Table 1: Values of $\theta_{b}$ and $\theta_{b}^{\prime}$ in $(6)$ for $b \leq 16$. Exponent pairs are given in terms of $A$ - and $B$-processes [6, Th. 2.12, 2.13]. We abbreviate $\mathcal{B}:=$ $B A$. Here $I=(0,1)$ and $H=(32 / 205+\varepsilon, 269 / 410+\varepsilon)$ is Huxley exponent pair from [4].

Lemma 3. Let $K \in \mathbf{N}, J \in \mathbf{N} \cup\{0\}, m_{1} \leq \cdots \leq m_{K}, n_{1} \leq \cdots \leq n_{J}$, where all $m_{k}, n_{j} \in \mathbf{N}$, and suppose that

$$
\sum_{n=1}^{\infty} \frac{a(n)}{n^{s}}=\frac{\zeta\left(m_{1} s\right) \cdots \zeta\left(m_{K} s\right)}{\zeta\left(n_{1} s\right) \cdots \zeta\left(n_{J} s\right)} .
$$

Let $\alpha=K-1 /\left(2 \sum_{k=1}^{K} m_{k}\right)$. If $1 / \alpha<2 n_{j}$ for all $j=1, \ldots, J$ then for arbitrary $H(x)$ of the form $H(x)=\sum_{i=1}^{I} x^{\beta_{i}} P_{i}(\log x), \beta_{i} \in \mathbf{C}, \alpha<\Re \beta_{i} \leq 1, P_{i}$ are polynomials, we have $\sum_{n \leq x} a_{n}=H(x)+\Omega\left(x^{\alpha}\right)$.

Proof. This is a simplified version of [7, Th. 2].

\section{Asymptotic Properties of $\sum \tau^{(r)}(n)$}

\section{Lemma 4.}

$$
\sum_{n=1}^{\infty} \frac{\tau^{(r)}(n)}{n^{s}}=\frac{\zeta(s) \zeta((r+1) s)}{\zeta((2 r+2) s)}, \quad \sigma>1
$$


Proof. Let us transform Bell series for $\tau^{(r)}$ :

$$
\begin{aligned}
\tau_{p}^{(r)}(x)=\sum_{k=0}^{\infty} \tau^{(r)}\left(p^{k}\right) x^{k} & =\sum_{k=0}^{r} x^{k}+2 \sum_{k>r} x^{k}=\sum_{k=0}^{\infty} x^{k}+\sum_{k>r} x^{k}= \\
=\left(1+x^{r+1}\right) \sum_{k=0}^{\infty} x^{k} & =\frac{1+x^{r+1}}{1-x}=\frac{1-x^{2 r+2}}{(1-x)\left(1-x^{r+1}\right)}
\end{aligned}
$$

Identity $\sum_{n=1}^{\infty} \frac{\tau^{(r)}(n)}{n^{s}}=\prod_{p} \tau_{p}^{(r)}\left(p^{-s}\right)$ completes the proof.

It follows from (7) that

$$
\tau^{(r)}=\tau(1, r+1 ; \cdot) \star \mu_{2 r+2}
$$

Theorem 5. If $\Delta$ is estimated as in (6) then for $r>0$

$$
\sum_{n \leq x} \tau^{(r)}(n)=A x+B x^{1 /(r+1)}+\mathcal{E}_{r+1}(x),
$$

where $\mathcal{E}_{r}(x)=O\left(x^{\max \left(\theta_{r}, 1 / 2 r\right)} \log ^{\theta_{r}^{\prime}} x\right)$, constants $A$ and $B$ are specified below in $(9)$.

Proof. Taking into account (8) we have for $r>0$

$$
\begin{aligned}
& \sum_{n \leq x} \tau^{(r)}(n)=\sum_{n \leq x} \mu_{2 r+2}(n) T(1, r+1 ; x / n)= \\
&=\zeta(r+1) x \sum_{n \leq x} \frac{\mu_{2 r+2}(n)}{n}+\zeta(1 /(r+1)) x^{1 /(r+1)} \sum_{n \leq x} \frac{\mu_{2 r+2}(n)}{n^{1 /(r+1)}}+ \\
&+\sum_{n \leq x} \mu_{2 r+2}(n) \Delta(1, r+1, x / n) .
\end{aligned}
$$

But for $s \geq 1 / k$

$$
\sum_{n \leq x} \frac{\mu_{k}(n)}{n^{s}}=\frac{1}{\zeta(k s)}-\sum_{n>x} \frac{\mu_{k}(n)}{n^{s}}=\frac{1}{\zeta(k s)}+O\left(x^{1 / k-s}\right)
$$

and

$$
\sum_{n \leq x} \mu_{2 k}(n) \Delta(1, k, x / n)=\sum_{n \leq x^{1 / 2 k}} \mu(n) \Delta\left(1, k, x / n^{2 k}\right) \ll
$$




$$
\ll \sum_{n \leq x^{1 / 2 k}}\left(\frac{x}{n^{2 k}}\right)^{\theta_{k}} \log ^{\theta_{k}^{\prime}} x \ll x^{\max \left(\theta_{k}, 1 / 2 k\right)} \log ^{\theta_{k}^{\prime}} x
$$

So

$$
\sum_{n \leq x} \tau^{(r)}(n)=\frac{\zeta(r+1)}{\zeta(2 r+2)} x+\frac{\zeta\left(\frac{1}{r+1}\right)}{\zeta(2)} x^{\frac{1}{r+1}}+E_{r+1}(x)
$$

Lemma 6. Let $r>0, x^{\varepsilon} \leq y \leq x^{1 / 2 r}$. Then under $R H$ we have

$$
\mathcal{E}_{r}(x)=\sum_{n \leq y} \mu(n) \Delta\left(1, r, x / n^{2 r}\right)+O\left(x^{1 / 2+\varepsilon} y^{1 / 2-r}+x^{\varepsilon}\right) .
$$

Proof. We follow the approach of Montgomery and Vaughan (see [9] or [1]).

First of all consider $g_{y}(s)=1 / \zeta(s)-\sum_{d \leq y} \mu(d) d^{-s}$. Then for $\sigma>1$ we have $g_{y}(s)=\sum_{d>y} \mu(d) d^{-s}$. Assuming $\mathrm{RH}$ we have by [12, Th. 14.25]

$$
\sum_{d \leq y} \frac{\mu(d)}{d^{s}}=\zeta^{-1}(s)+O\left(y^{1 / 2-\sigma+\varepsilon}\left(|t|^{\varepsilon}+1\right)\right) \quad \text { for } \sigma>1 / 2+\varepsilon,
$$

so

$$
g_{y}(s) \ll y^{1 / 2-\sigma+\varepsilon}\left(|t|^{\varepsilon}+1\right) \quad \text { for } \sigma>1 / 2+\varepsilon .
$$

Now let us split $\sum_{n \leq x} \tau^{(r-1)}(n)$ into two parts:

$$
\sum_{n \leq x} \tau^{(r-1)}(n)=\sum_{d^{2 r} \leq x} \mu(d) T\left(1, r ; x / d^{2 r}\right)=S_{1}+S_{2},
$$

where

$$
\begin{aligned}
S_{1}:=\sum_{d \leq y} \mu(d) T\left(1, r ; x / d^{2 r}\right) & =\zeta(r) x \sum_{d \leq y} \frac{\mu(d)}{d^{2 r}}+ \\
& +\zeta(1 / r) x^{1 / r} \sum_{d \leq y} \frac{\mu(d)}{d^{2}}+\sum_{d \leq y} \mu(d) \Delta\left(1, r ; x / d^{2 r}\right)
\end{aligned}
$$

and $S_{2}$ is the rest of $\sum_{n \leq x} \tau^{(r-1)}(n)$. We note that under RH by taking into account $y \leq x^{1 / 2 r}$ we have

$$
x^{1 / r} \sum_{d>y} \frac{\mu(d)}{d^{2}} \ll x^{1 / r} y^{-3 / 2+\varepsilon} \ll x^{1 / 2} y^{1 / 2-r+\varepsilon}
$$


and so

$$
x^{1 / r} \sum_{d \leq y} \frac{\mu(d)}{d^{2}}=\frac{x^{1 / r}}{\zeta(2)}+O\left(x^{1 / 2} y^{1 / 2-r+\varepsilon}\right) .
$$

Next, let $h_{y}(s):=\zeta(s) \zeta(r s) g_{y}(2 r s) x^{s} s^{-1}$. Then by Perron formula with $c=1+\varepsilon, T=x^{2}$ one can estimate

$$
S_{2}=\frac{1}{2 \pi i} \int_{1+\varepsilon-i x^{2}}^{1+\varepsilon+i x^{2}} h_{y}(s) d s+O\left(x^{\varepsilon}\right) .
$$

By moving line of integration to $\left[1 / 2+\varepsilon-i x^{2}, 1 / 2+\varepsilon+i x^{2}\right]$ we obtain

$$
S_{2}=\underset{s=1}{\operatorname{res}} h(s)+O\left(I_{1}+I_{2}+I_{3}\right),
$$

where

$$
I_{1}=\int_{1+\varepsilon-i x^{2}}^{1 / 2+\varepsilon-i x^{2}} h(s) d s, I_{2}=\int_{1 / 2+\varepsilon-i x^{2}}^{1 / 2+\varepsilon+i x^{2}} h(s) d s, I_{3}=\int_{1 / 2+\varepsilon-i x^{2}}^{1+\varepsilon-i x^{2}} h(s) d s .
$$

Due to (11) and estimates of $\zeta$ under RH we have

$$
\begin{array}{cl}
g_{y}(2 r s) \ll y^{1 / 2-r}\left(|t|^{\varepsilon}+1\right) & \text { for } \sigma>1 / 2+\varepsilon, \\
h(s) \ll y^{1 / 2-r}\left(|t|^{\varepsilon}+1\right) x^{s} s^{-1} & \text { for } \sigma>1 / 2+\varepsilon,
\end{array}
$$

and

$$
\begin{gathered}
I_{1,3} \ll y^{1 / 2-r+\varepsilon} \max _{\sigma \in[1 / 2+\varepsilon, 1+\varepsilon]} x^{\sigma-2} \ll y^{1 / 2-r+\varepsilon}, \\
I_{2} \ll y^{1 / 2-r+\varepsilon} \int_{1}^{x^{2}} x^{1 / 2} t^{-1} d t \ll y^{1 / 2-r+\varepsilon} x^{1 / 2+\varepsilon} .
\end{gathered}
$$

Identity

$$
\underset{s=1}{\operatorname{res}} h(s)=\zeta(r) x \sum_{d>y} \frac{\mu(d)}{d^{2 r}}
$$

completes the proof.

Theorem 7. If $\Delta$ is estimated as in (6) and $\theta_{r}<1 / 2 r$ then under $R H$ we get $\mathcal{E}_{r}(x)=O\left(x^{\alpha}\right)$, where $\alpha=\left(1-\theta_{r}\right) /\left(2 r+1-4 r \theta_{r}\right)$.

Proof. Let us start with (10):

$$
\mathcal{E}_{r}(x)=\sum_{n \leq y} \mu(n) \Delta\left(1, r, x / n^{2 r}\right)+O\left(x^{1 / 2+\varepsilon} y^{1 / 2-r}+x^{\varepsilon}\right) \ll
$$




$$
\begin{aligned}
& \ll \sum_{n \leq y}\left(\frac{x}{n^{2 r}}\right)^{\theta_{r}+\varepsilon}+O\left(x^{1 / 2+\varepsilon} y^{1 / 2-r}+x^{\varepsilon}\right) \ll \\
& \ll x^{\varepsilon}\left(x^{\theta_{r}}\left(1+y^{1-2 r \theta_{r}}\right)+x^{1 / 2} y^{1 / 2-r}+1\right) .
\end{aligned}
$$

If $\theta_{r}<1 / 2 r$ then $\mathcal{E}_{r}(x) \ll x^{\varepsilon}\left(x^{\theta_{r}} y^{1-2 r \theta_{r}}+x^{1 / 2} y^{1 / 2-r}\right)$. Choice $y=x^{\beta}$, where $\beta=\left(1-2 \theta_{r}\right) /\left(2 r+1-4 r \theta_{r}\right)$, accomplishes the proof.

For the values of $\theta_{b}$ from Table 1 we have

$$
\max \left(\theta_{r}, 1 / 2 r\right)= \begin{cases}1 / 2 r, & r \leq 2 \\ \theta_{r}, & r>2\end{cases}
$$

So currently the only non-trivial case of the previous theorem is an estimation for $\tau^{(1)} \equiv \tau^{(e) s}$. We get under assumption of RH that

$$
\begin{gathered}
\sum_{n \leq x} \tau^{(1)}(n)=\frac{\zeta(2)}{\zeta(4)} x+\frac{\zeta(1 / 2)}{\zeta(2)} x^{1 / 2}+O\left(x^{\alpha+\varepsilon}\right), \\
\alpha=\frac{1-\theta_{2}}{5-8 \theta_{2}}=\frac{3728}{15469} \approx 0.241<1 / 4 .
\end{gathered}
$$

Theorem 8. $\mathcal{E}_{r}(x)=\Omega\left(x^{1 /(2 r+2)}\right)$.

Proof. The statement is implied by the substitution $m_{1}=1, m_{2}=r$, $n_{1}=2 r$ into Lemma 3 . The choice of parameters plainly follows from (7). We obtain $\alpha=1 /(2 r+2)$, which is an exponent in the required $\Omega$-term.

\section{Asymptotic Properties of $\sum \sigma^{(r)}$}

\section{Lemma 9.}

$$
\sum_{n=1}^{\infty} \frac{\sigma^{(r)}(n)}{n^{s}}=\frac{\zeta(s-1) \zeta((r+1) s-r)}{\zeta((r+2) s-r-1)} H_{r}(s), \quad \sigma>2
$$

where Dirichlet series $H_{r}(s)$ converges absolutely for $\sigma>(2 r+2) /(2 r+3)$.

Proof. Consider Bell series for $\sigma^{(r)}$ :

$$
\sigma_{p}^{(r)}(x):=\sum_{k=0}^{\infty} \sigma^{(r)}\left(p^{k}\right) x^{k}=\sum_{k=0}^{r} p^{k} x^{k}+\sum_{k>r}\left(p^{r}+p^{k}\right) x^{k}=
$$




$$
=\frac{1}{1-p x}+\frac{p^{r} x^{r+1}}{1-x}
$$

Then

$$
\frac{(1-p x)\left(1-p^{r} x^{r+1}\right)}{1-p^{r+1} x^{r+2}} \sigma_{p}^{(e) s}(x)=1+\frac{p^{r} x^{r+2}(1-p x)\left(1-p^{r} x^{r}\right)}{(1-x)\left(1-p^{r+1} x^{r+2}\right)}:=h_{p}(x) .
$$

For $\sigma>1$ we have $h_{p}\left(p^{-s}\right) \ll p^{-2}$. For $1 \geq \sigma \geq(2 r+2) /(2 r+3)+\varepsilon$ we have $h_{p}\left(p^{-s}\right) \ll p^{2 r+1-(2 r+3) s} \ll p^{-1-\varepsilon}$. Now (12) follows from the representation $\sum_{n=1}^{\infty} \frac{\sigma^{(r)}(n)}{n^{s}}=\prod_{p} \sigma_{p}^{(r)}\left(p^{-s}\right)$.

\section{Theorem 10.}

$$
\sum_{n \leq x} \sigma^{(r)}(n)=D x^{2}+O\left(x \log ^{5 / 3} x\right), \quad D=\frac{\zeta(r+2) H_{r}(2)}{2 \zeta(r+3)} .
$$

Proof. For a fixed $r$ let $z(n)$ be the coefficient at $n^{-s}$ of the Dirichlet series

$$
\frac{\zeta(s-1) \zeta((r+1) s-r)}{\zeta((r+2) s-r-1)}
$$

and let $h(n)$ be the coefficient of the Dirichlet series $H_{r}(s)$. It follows from (12) that $\sigma^{(r)}=z \star h$. One can verify that

$$
z(n)=\sum_{a b^{r+1} c^{r+2}=n} a b^{r} c^{r+1} \mu(c) .
$$

Taking into account Lemma 2 with $(\alpha, \beta)=(r+1, r)$ we obtain

$$
\begin{aligned}
\sum_{n \leq x} z(n)=\sum_{c \leq x^{1 /(r+2)}} c^{r+1} \mu(c)\left(\frac{\zeta(r+2)}{2} \frac{x^{2}}{c^{2 r+4}}+\right. & \left.O\left(x c^{-r-2} \log ^{2 / 3} x\right)\right)= \\
& =\frac{\zeta(r+2)}{2 \zeta(r+3)} x^{2}+O\left(x \log ^{5 / 3} x\right) .
\end{aligned}
$$

Now

$$
\begin{aligned}
\sum_{n \leq x} \sigma^{(r)}(n)=\sum_{n \leq x} h(n) & \left(\frac{\zeta(r+2)}{2 \zeta(r+3)} \frac{x^{2}}{n^{2}}+O\left(\frac{x}{n} \log ^{5 / 3} x\right)\right)= \\
& =\frac{\zeta(r+2)}{2 \zeta(r+3)} x^{2} \sum_{n \leq x} \frac{h(n)}{n^{2}}+O\left(x \log ^{5 / 3} x \sum_{n \leq x} \frac{h(n)}{n}\right) .
\end{aligned}
$$


But $H_{r}(s)$ converges absolutely at $\sigma \geq(2 r+2) /(2 r+3)+\varepsilon$, so $\sum_{n \leq x} \frac{h(n)}{n} \ll O(1)$ and

$$
\sum_{n \leq x} \frac{h(n)}{n^{2}}=H_{r}(2)-\sum_{n>x} \frac{h(n)}{n^{2}}=H_{r}(2)+O\left(x^{-(2 r+4) /(2 r+3)+\varepsilon}\right) .
$$

Theorem 11. For a fixed $r>0 \sum_{n \leq x} \sigma^{(r)}(n)=D x^{2}+\Omega_{ \pm}(x \log x)$.

Proof. The proof almost replicates the proof of [10, Th. 3] with following changes (in notations of [10]):

$$
\begin{gathered}
\kappa(n):=\frac{\sigma^{(r)}(n)}{n}, \quad \sum_{n=1}^{\infty} \frac{\kappa(n)}{n^{s}}=\frac{\zeta(s) \zeta((r+1) s+1)}{\zeta((r+2) s+1)} H_{r}(s+1), \\
v:=\mu \star \kappa, \quad \sum_{n=1}^{\infty} \frac{v(n)}{n^{s}}=\frac{\zeta((r+1) s+1)}{\zeta((r+2) s+1)} H_{r}(s+1), \\
v\left(p^{a}\right)=\frac{\sigma^{(r)}\left(p^{a}\right)}{p^{a}}-\frac{\sigma^{(r)}\left(p^{a-1}\right)}{p^{a-1}}= \begin{cases}0, & a \leq r+1, \\
1 / p, & a=r+1, \\
p^{r-a}-p^{r-a+1}, & a>r+1 .\end{cases}
\end{gathered}
$$

We take $m:=\log ^{1 /(4 r+4)} x$ and $A:=\prod_{p \leq m} p^{r+1} \sim e^{(r+1) m} \sim \exp \left(\log ^{1 / 4} x\right)$, then

$$
G=\sum_{k \leq u(x)} \frac{v(k)}{k} \operatorname{gcd}(A, k)=\sum_{n^{r+1} \mid A} v\left(n^{r+1}\right) \sum_{k \leq u(x) / n^{r+1}}^{*} \frac{v\left(n^{r+1} k\right)}{v\left(n^{r+1}\right) k} .
$$

Here $\sum_{k}^{*}$ means summation over $k$ such that for every $p \mid k$ we have $p \mid n$ or $p \bigvee A$. Taking into account $v\left(p^{r+1}\right)=1 / p$ we get

$$
\begin{aligned}
& G=\sum_{n^{r+1} \mid A} v\left(n^{r+1}\right) \sum_{k \geq 1}^{*} \frac{v\left(n^{r+1} k\right)}{v\left(n^{r+1}\right) k}+o(1)= \\
& \quad=\sum_{n^{r+1} \mid A} v\left(n^{r+1}\right) \prod_{p \mid n}\left(1+\sum_{\nu \geq r+2} \frac{v\left(p^{\nu}\right)}{p^{\nu-r-2}}\right) \prod_{p>m}\left(1+\sum_{\nu \geq r+1} \frac{v\left(p^{\nu}\right)}{p^{\nu}}\right)+o(1) .
\end{aligned}
$$

Since $\left|v\left(p^{\nu}\right)\right| \leq 1 / p$ we obtain $\sum_{\nu \geq r+1} v\left(p^{\nu}\right) p^{-\nu} \ll p^{-r-2}$. Since $v\left(n^{r+1}\right)=1 / n$ for $n^{r+1} \mid A$ and $\log m \asymp \log x$ we have 


$$
\begin{aligned}
G=(1+o(1)) \sum_{n^{r+1} \mid A} \frac{1}{n} \prod_{p \mid n}(1 & \left.+\sum_{\nu \geq r+2} \frac{v\left(p^{\nu}\right)}{p^{\nu-r-2}}\right)= \\
& =(1+o(1)) \prod_{p \leq m}\left(1+\frac{1}{p}+\sum_{\nu \geq r+2} \frac{v\left(p^{\nu}\right)}{p^{\nu-r-1}}\right)
\end{aligned}
$$

But $v\left(p^{\nu}\right) \geq 1 / 2 p^{\nu-r-1}$ for $a \geq r+2$. So

$$
\sum_{\nu \geq r+2} v\left(p^{\nu}\right) p^{-\nu+r+1} \geq \sum_{\nu \geq r+2} p^{2(-\nu+r+1)} / 2 \geq p^{-2} / 2 .
$$

Hence

$$
G \gg \prod_{p \leq m}\left(1+p^{-1}+p^{-2} / 2\right) \gg \prod_{p \leq m}\left(1+p^{-1}\right) \gg \log m \gg \log x .
$$

\section{References}

[1] X. Cao, W. Zhai, Some arithmetic functions involving exponential divisors, J. Integer Seq., 13, No. 3 (2010), 10.3.7.

[2] A. A. Gioia, A. M. Vaidya, The number of squarefree divisors of an integer, Duke Math. J., 33, No. 4 (1966), 797-799.

[3] S. W. Graham, G. Kolesnik, On the difference between consecutive squarefree integers, Acta Arith., 49, No. 5 (1988), 435-447.

[4] M. N. Huxley, Exponential sums and the Riemann zeta function V, Proc. Lond. Math. Soc., 90, No. 1 (2005), 1-41.

[5] A. Ivić, The Riemann zeta-function, Dover Publications, USA (2003).

[6] E. Krätzel, Lattice points, Kluwer, Netherlands (1988).

[7] M. Kühleitner, W. G. Nowak, An omega theorem for a class of arithmetic functions, Math. Nachr., 165, No. 1 (1994), 79-98.

[8] N. Minculete, Contribuţii la studiul proprietătilor analitice ale functiilor

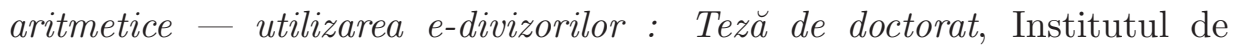
Matematică "Simion Stoilow", Romania (2011). 
[9] H. L. Montgomery, R. C. Vaughan, On the distribution of square-free numbers, In: Recent progress in analytic number theory, Academic Press, USA (1981), 247-256.

[10] Y.-F. S. Pétermann, J. Wu, On the sum of exponential divisors of an integer, Acta Math. Hung., 77, No. 1-2 (1997), 159-175.

[11] R. Sita Rama Chandra Rao, D. Suryanarayana, On $\sum_{n \leq x} \sigma^{*}(n)$ and $\sum_{n \leq x} \phi^{*}(n)$, Proc. Am. Math. Soc., 41, No. 1 (1973), 61-66.

[12] E. C. Titchmarsh, The theory of the Riemann zeta-function, Oxford University Press, UK (1986). 